\title{
Applying micro-computed tomography for porosity analysis of polypropylene modified with microspheres
}

\author{
Piotr Szewczykowski1,** \\ ${ }^{1}$ UTP University of Science and Technology, Faculty of M echanical Engineering, AI. prof. S. \\ K aliskiego 7, 85-796 Bydgoszcz, Poland
}

\begin{abstract}
Porosity of polypropylene samples was investigated by applying $X$-ray micro-computed tomography (micro-CT), which is getting more and more popular as a non-destructive method. M icrospheres were applied as a blowing agent at three concentrations: $3 \%, 6 \%$ and $9 \%$ by weight. Tensile testing specimens were obtained by injection molding technology and its central, measuring part were examined by micro - CT. Results were compared to porosity calculated based on difference in porous and solid material density. Pore size distribution curves were discussed as well.
\end{abstract}

\section{Introduction}

$\mathrm{X}$-ray micro-computed tomography (micro-CT) is a non-invasive method that allows examining the internal structure of the material. Thanks to the absorption of X-ray waves by materials of different densities, we can obtain contrast and observe an internal structure. Especially polymeric porous structures with pores filled with air or other gas can seen very well by this method. Micro-computed tomography is also applied in such areas as medicine [1], automotive [1], food [3], pharmacy [4], 3D printing etc. In order to observe an internal structure and pores of given sample scanning electron microscopy can be applied, but only a surface revealed after breaking the sample can be observed. Another advantage of micro$\mathrm{CT}$ is no need for sample preparation. Huge amount of generated data and as a consequence necessity for high computing power can be seen as disadvantage, not mentioning price of individual apparatus. Possibility to non-destructive analysis of sample within a given volume, together with wide spectrum of visualization internal structures or cross-sections makes this method more and more popular in scientific research.

The aim of this work is present one of these possibilities to calculate samples porosity and pore size distribution of polypropylene modified with microspheres.

\section{Methodology}

The moldings in the form of tensile test specimen made of Moplen HP $500 \mathrm{~N}$ polypropylene by Lyondell Basell were obtained by ENGEL e-Victory 310/110 hybrid injection molding machine. The porous material was obtained by adding Akzo Nobel 930 MB 120 microspheres in amount of 3, 6 and $9 \%$ by weight. The injection mold was thermostated at $30^{\circ} \mathrm{C}$. The temperature of the nozzle, heating zones III, II, I and the feed zone were: $200{ }^{\circ} \mathrm{C}, 200{ }^{\circ} \mathrm{C}, 180{ }^{\circ} \mathrm{C}, 170{ }^{\circ} \mathrm{C}$ and $40{ }^{\circ} \mathrm{C}$, respectively. Bars of approx. 50 $\mathrm{mm}$ long were cut from the central measuring section of injection molded tensile test specimens and analyzed with x-ray micro-computed tomograph SkyScan 1272 from Bruker. Image pixel size was $3,5 \mu \mathrm{m}$, source voltage $40 \mathrm{kV}$ and rotation step $0,3 \mathrm{deg}$. The structure reconstruction was obtained with the use of Nrecon program. CTan program was

\footnotetext{
* Corresponding author: piotr.szewczykowski@utp.edu.pl
} 
applied for data analysis and porosity calculations. Rectangular region of interest (ROI) was applied, so calculated porosity did not included the whole sample cross-section (Figure 1). Samples porosity was recalculated later on accordingly to the whole cross-section. CTVox program was used for three-dimensional sample cross-section visualization.

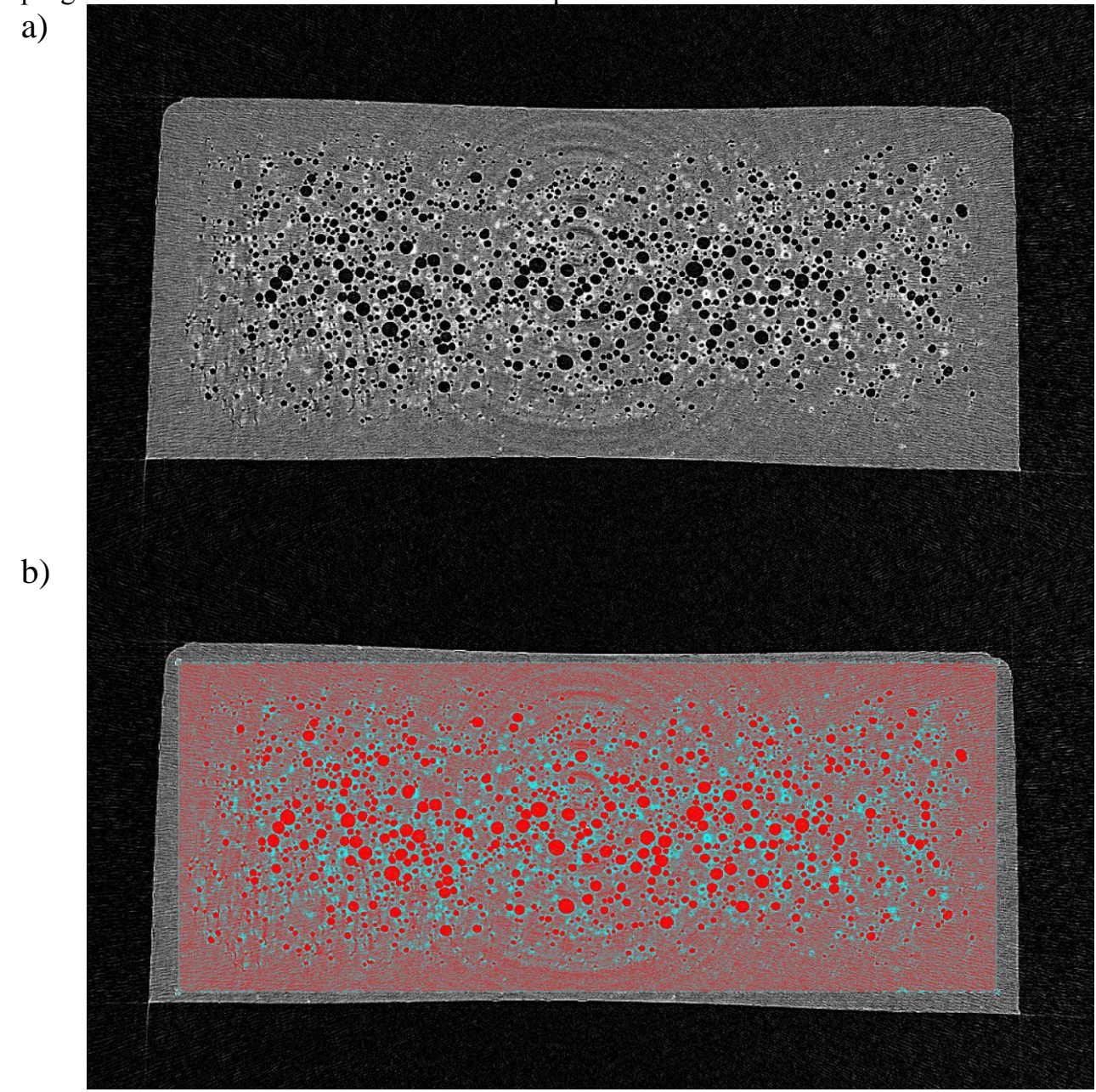

Fig. 1. a) A slice (in the middle of analyzed length) of sample with $3 \%$ microspheres. Picture after reconstruction is opened in CTAn program, b) A slice with marked region of interest (ROI) which was analyzed in next steps.

Samples were investigated with S3000N scanning electron microscope by Hitachi as well. Samples were sputter coated with gold - palladium by Quorum Technologies sputter coater SC7620. Picture registered by SEM was analyzed by using Image J software to estimate an average pore size.

\section{Results and discussion}

The differences in amount of pores and its size between samples with $3 \%, 6 \%$ and $9 \%$ added microspheres can be already observed within analysed region of interest after binary conversion. Additional despeckle operation was applied in order to get rid of a noise like small objects within a given pore (Figure 2). 


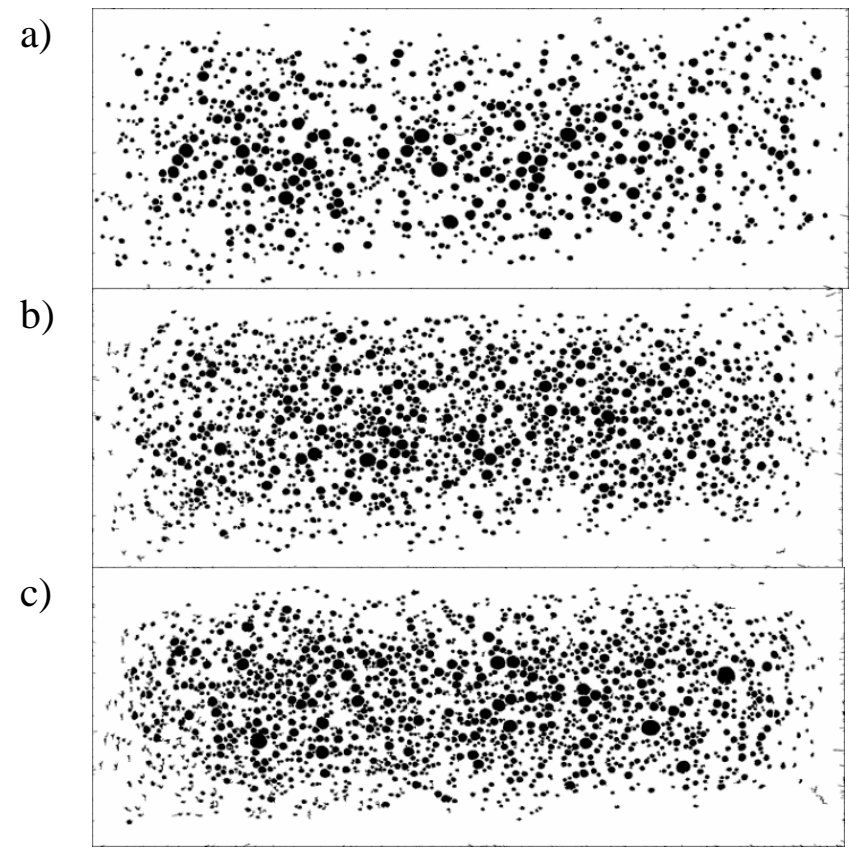

Fig. 2. Single slices of polypropylene samples with $3 \%, 6 \%$ and $9 \%$ of microspheres added (Fig. $2 a$, Fig. $2 \mathrm{~b}$ and Fig.2c respectively) after binary conversion and applying a despeckle operation in CTAn program.

Pore size distribution for each sample was obtained after 3D analysis of pores within sample volume by CTAn program. Results are plotted in Figure 3. The maximum volume within samples is occupied by pores in range of $136-143 \mu \mathrm{m}, 87-94 \mu \mathrm{m}$ and $73-80 \mu \mathrm{m}$ for sample with $3 \%, 6 \%$ and $9 \%$ microspheres respectively. Higher amount of microspheres added causes higher concentration pores so its growth limits the maximum pore size.

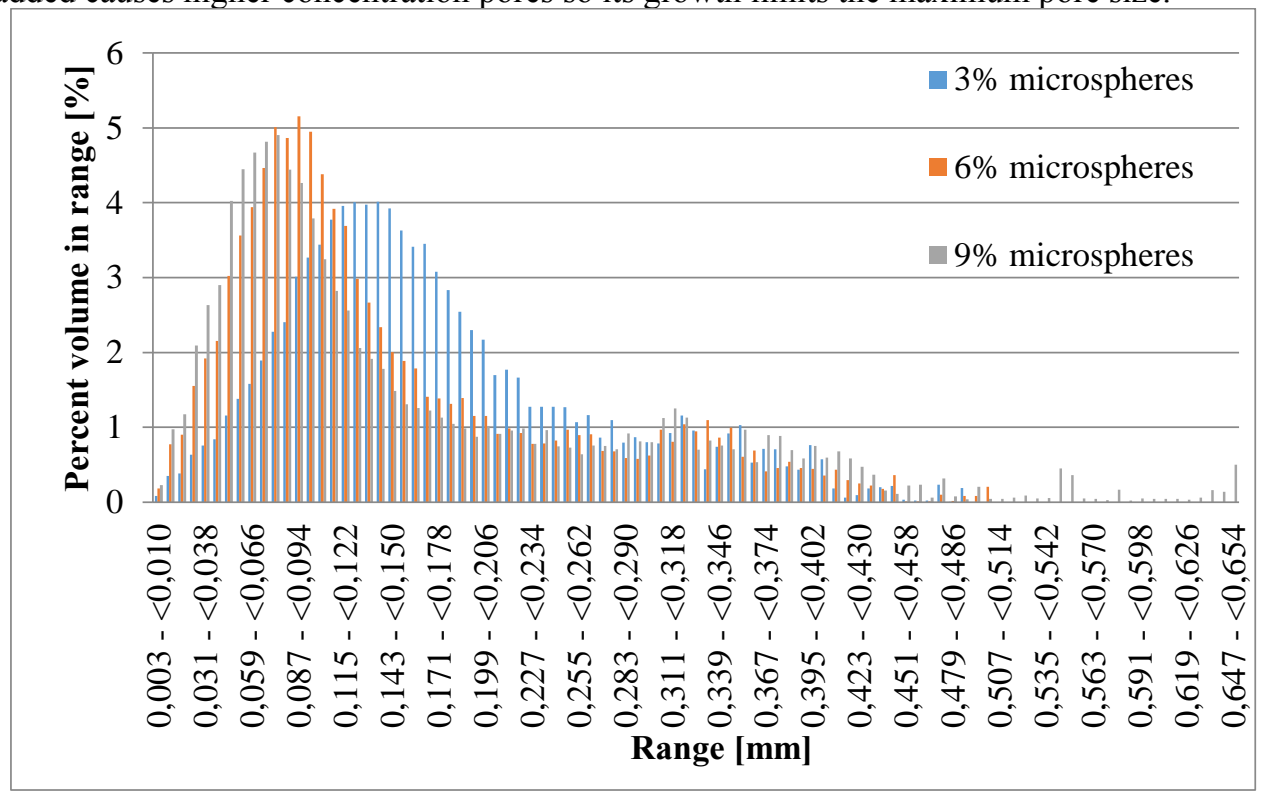

Fig. 3. Percentage volume of pores in a given range for polypropylene samples with $3 \%, 6 \%$ and $9 \%$ microspheres added (blue, orange and grey bars respectively). 
Porosity was calculated for each sample based on its density firstly. Density was calculated based on mass and volume measurements, while porosity was considered in reference to a solid polypropylene samples. Based on this method sample porosity increased by 2,81 after adding $3 \%$ microspheres, while there so no porosity difference observed between samples with $6 \%$ and $9 \%$. Similar tendency show the CTAn program analysis. Porosity of PP with $6 \%$ microsphere is 3,37 higher comparing to sample with $3 \%$ of microspheres, while porosity difference between $6 \%$ and $9 \%$ microspheres sample is only 0,44 . Porosity values are higher for CTAn analysis comparing to measurements based on density. This is caused by ROI no covering sample cross-section precisely.

There is an option of choosing an interpolated shape of ROI and fit it by hand. The risk is that in case the sample shape changes in following slices the ROI can extend the sample edge. The area outside the sample can be interpreted as a void, which increase a mistake of final porosity results. Therefore it can be reasonable to choose smaller ROI within a sample cross-section, except that porosity results may be overestimated. Results obtained by CTAn program were recalculated to the whole sample cross-section. Difference in samples and ROI area was calculated and porosity results were corrected. After this operation results from CTAn are close to those obtained from density calculations but still higher.

Table 1. Porosity of samples with $3 \%, 6 \%$ and $9 \%$ of added microsphere calculated from mass and volume measurements (second column), obtained by analysis with CTAn program (third column) and after correction referring to ROI and original sample cross-section (last column).

\begin{tabular}{|c|c|c|c|}
\hline $\begin{array}{c}\text { Percentage } \\
\text { of microspheres } \\
\text { added }\end{array}$ & $\begin{array}{c}\text { Porosity } \\
\text { (mass and } \\
\text { volume measured) } \\
{[\%]}\end{array}$ & $\begin{array}{c}\text { Porosity } \\
\text { (by CTAn) } \\
{[\%]}\end{array}$ & $\begin{array}{c}\text { Porosity } \\
\text { (by CTAn) } \\
\text { corrected } \\
{[\%]}\end{array}$ \\
\hline $3 \%$ & 10,04 & 13,88 & 11,60 \\
\hline $6 \%$ & 12,85 & 17,25 & 14,41 \\
\hline $9 \%$ & 12,85 & 17,69 & 14,78 \\
\hline
\end{tabular}

CTVox program was applied to visualise sample cross-sections in 3D. Visualisations of samples with $3 \%, 6 \%$ and $9 \%$ of microspheres are presented in Figure 4 .

a)

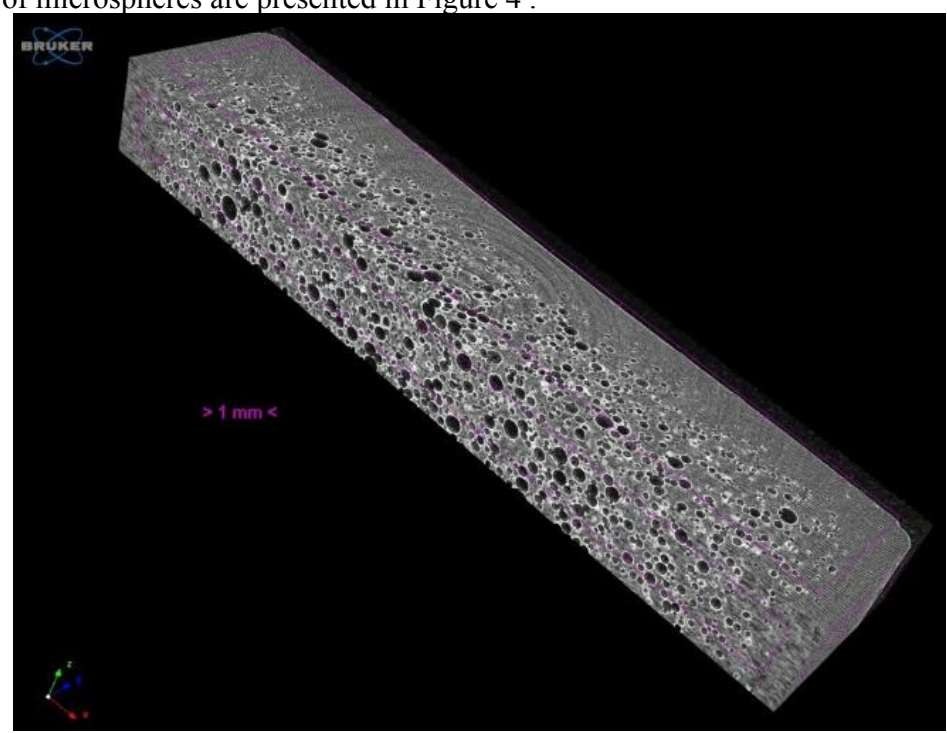


b)

c)

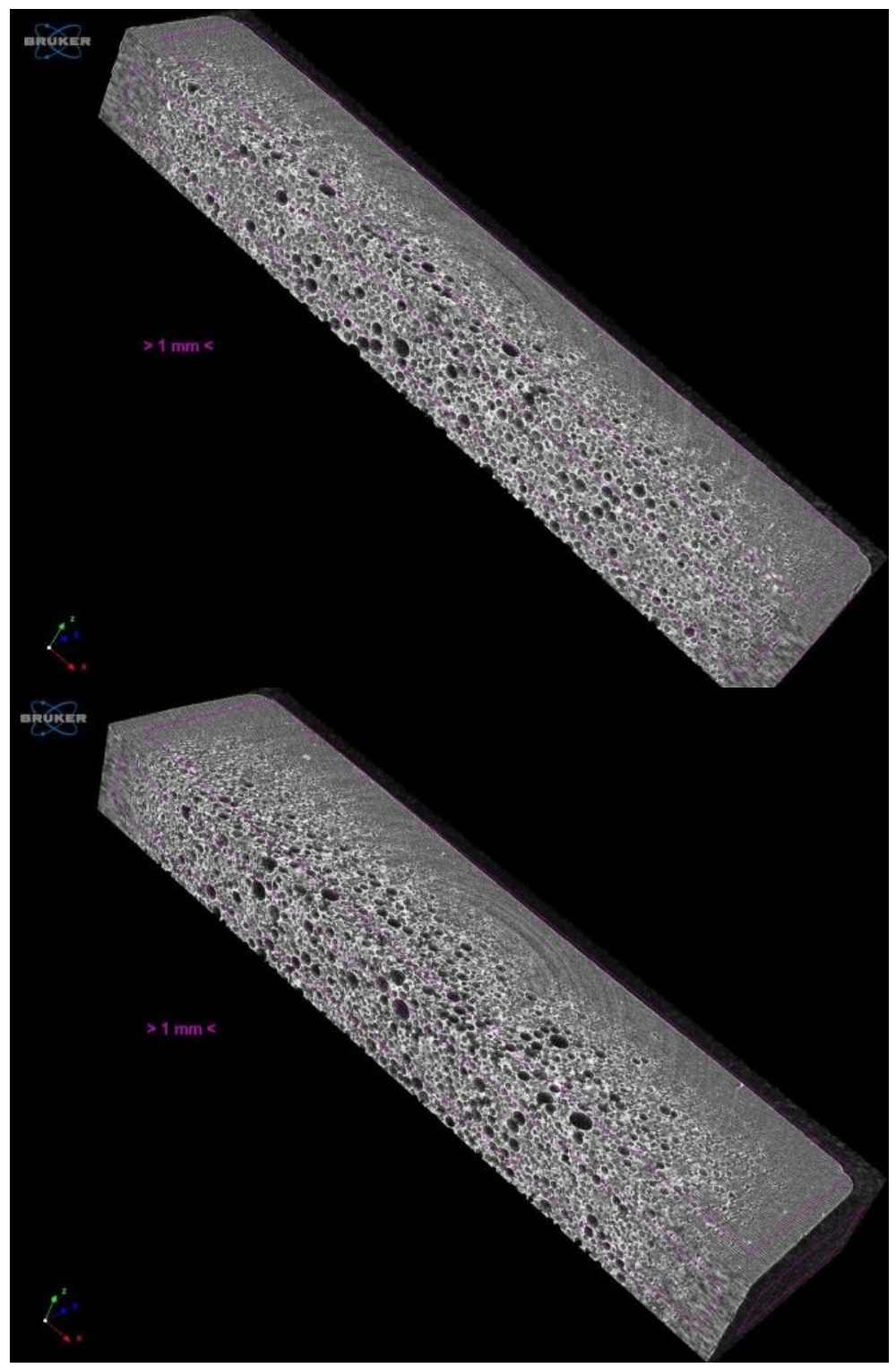

Fig. 4. Cross-section of analysed samples with $3 \%, 6 \%$ and $9 \%$ of microspheres added (Fig.4a, Fig. $4 \mathrm{~b}$ and Fig. $4 \mathrm{c}$ respectively) visualised in CTVox program.

Herewith one can observe a scanning electron microscopy picture of sample crosssection with $3 \%$ microspheres added. Image analysis was performed by using Image $\mathrm{J}$ program. The result of fitting the objects to cover areas identified as pores is presented in Figure 5. The average pore size after fitting 345 objects to the image is $90,8 \mu \mathrm{m}$ diameter, which is relatively lower comparing to micro-CT results. One of the reason of such a difference can be analysis of a single fracture surface (SEM) comparing to measumets within the whole volume (micro-CT) 
a)

b)

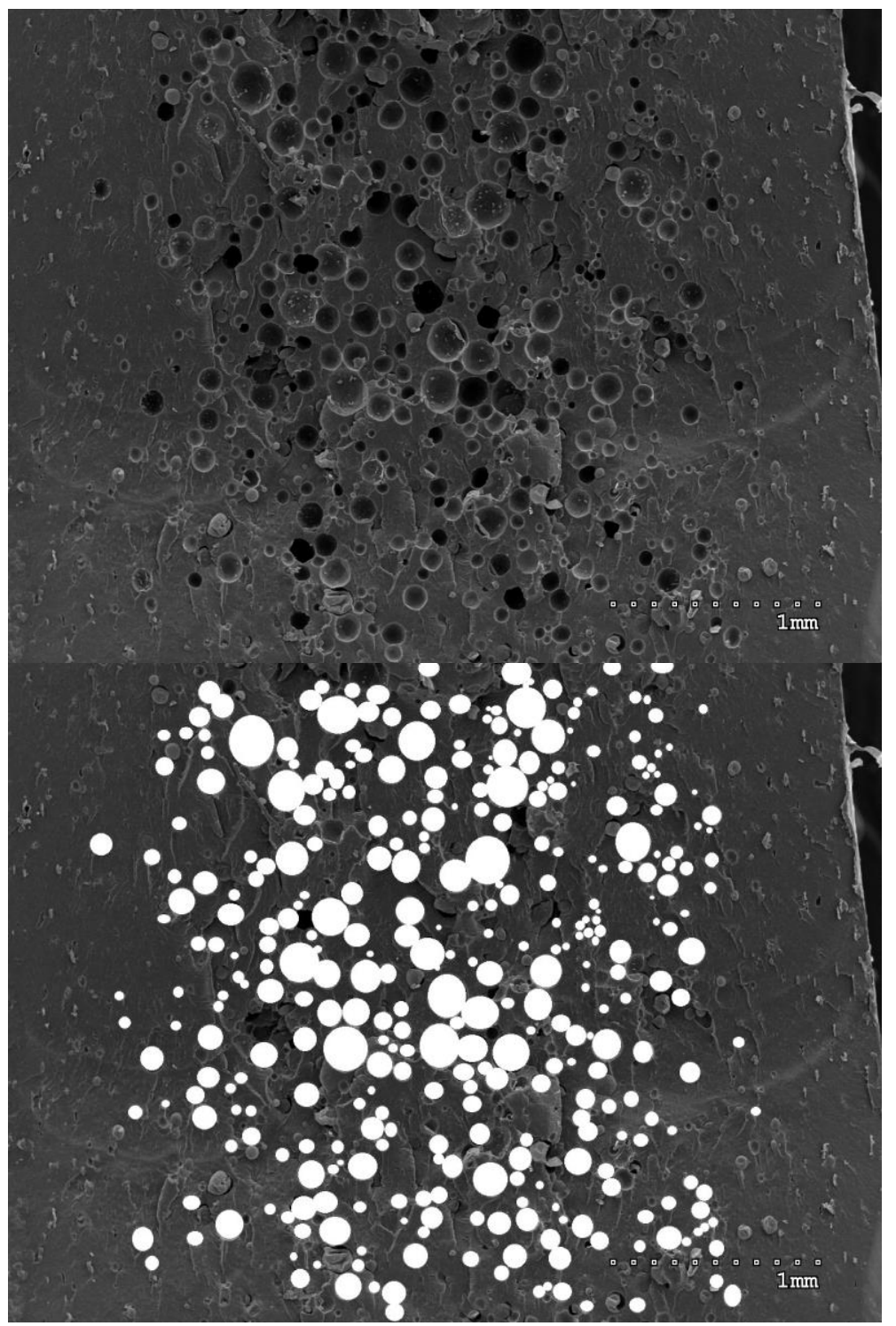

Fig. 5. a) Scanning electron microscopy picture of polypropylene sample cross-section with $3 \%$ microspheres added. Image registered at $20 \mathrm{kV}$ voltage and working distance $21.7 \mathrm{~mm}$ by secondary electrons detector. Sample was sputter coated with gold-palladium, b) picture after fitting objects in Image $\mathrm{J}$ picture software in order to calculate average pore size.

\section{Conclusions}

Micro-computed tomography is a very useful method to obtain porosity of polymeric samples. The difference between pore size and micro-tomography scanning resolution is high enough in case of applied microspheres, so sharp images and visualisations can be obtained. The highest increase in sample porosity was observed between samples with $3 \%$ and $6 \%$ microspheres added. 
The acknowledgements should be typed in 9-point Times, without title. The author would like to thank the COMEF COMEF Company (especially Piotr Czerwiński and Jakub Śluzar) for providing measuring time at SkyScan 1272 BRUKER micro-CT and for technical support. Thanks to Magdalena Gawlak from the Institute of Plant Protection - National Research Institute for scanning electron microscopy pictures.

\section{References}

1. D. Cvetkovic, et al. Calcified Tissue International , 107, 4 (2020)

2. D. Sykutera, P. Szewczykowski, M. Roch, L. Wajer, M. Grabowski, M. Bieliński Polimery, 63, 11-12 (2018)

3. L. Schoeman, P. Williams; A. du Plessis, et al. Trends in Food Science and Technology, 47 (2016)

4. $X Z$ Y in et al. Materials Science \& Engineering C-Materials for Biological A pplications, 116 (2020)

5. P.P. Szewczykowski, Ł. Skarżyński, Ł. Polimery, 64 (2019) 